\title{
Il y a 150 ans, les Bulletins et Mémoires de la Société d'Anthropologie de Paris publiaient les découvertes de Cro-Magnon
}

\author{
150 years ago, the Bulletins et Mémoires de la Société d'Anthropologie de Paris published \\ the discoveries of Cro-Magnon
}

\author{
A. Mounier · M. Gibert · G. Goude $\cdot$ F. Marchal $\cdot$ A. Thomas \\ C) Société d'Anthropologie de Paris et Lavoisier SAS 2018
}

Pour ce numéro d'octobre 2018, les Bulletins et Mémoires de la Société d'Anthropologie de Paris reviennent sur une histoire qui commence comme beaucoup de « faits préhistoriques » par un hasard heureux, qui vit des ouvriers mettre au jour ce qui deviendra l'un des symboles de l'archéologie et de la paléoanthropologie. Cent cinquante ans après l'annonce par le journal L'Echo de la Dordogne de la découverte de restes humains datant de "l'âge de la pierre » aux Eyzies-de-Tayac, cet événement, qui peut paraître anodin mais qui fascina le public à l'époque, s'impose toujours comme un moment fondateur de l'histoire de notre discipline.

Au-delà des enjeux politiques, qui transparaissent au travers de l'intérêt porté à la découverte par le ministre de l'Instruction publique [1], c'est la méthode de fouille et l'interprétation du site [2] qui interpellent par leur modernité et qui constituent l'un des premiers jalons fondateurs des sciences

\author{
A. Mounier \\ UMR 7194 HNHP, CNRS, PVD, MNHN, Musée de l'Homme, \\ Paris, France
}

Leverhulme Centre for Human Evolutionary Studies,

University of Cambridge, United Kingdom

M. Gibert

UMR 5288 AMIS, CNRS, Université Toulouse III-Paul Sabatier,

Toulouse France

G. Goude

Aix-Marseille université, CNRS, ministère de la Culture,

LAMPEA, Aix-en-Provence, France

F. Marchal

UMR 7268 ADES, Aix Marseille Université, CNRS, EFS,

Marseille, France

A. Thomas

Eco-anthropologie et Ethnobiologie (EAE), MNHN, CNRS,

Université Paris Diderot, Paris, France préhistoriques et paléoanthropologiques naissantes. Le site de Cro-Magnon va en effet bénéficier d'une attention toute particulière de la part du géologue Louis Lartet. Celui-ci va accéder au site quelques jours à peine après la découverte fortuite des ossements et va pouvoir entamer des fouilles « régulières et méthodiques » $[2: 341]$ d'un site archéologique relativement peu modifié. Son analyse [2] va permettre d'alimenter et de clore au moins momentanément, le débat sur l'antiquité de l'Homme, très vivace dans la seconde moitié du XIX ${ }^{\mathrm{e}}$ siècle, dans un contexte de publications polémiques et innovantes, qui voit notamment paraître le célèbre On the Origin of Species de Charles Darwin [3].

Ce contexte académique marque le début de la Préhistoire en tant que discipline théorique, puisqu'il ouvre la voie à la reconnaissance de l'ancienneté de l'Homme et de son histoire biologique commune avec l'ensemble du vivant. Cependant, si l'Histoire de l'Homme peut désormais être considérée avec sa dimension " antédiluvienne ", il n'existe aucune preuve de l'ancienneté réelle de l'humanité. C'est ce débat, qui va agiter la communauté scientifique pendant de nombreuses années [1], et que le site de Cro-Magnon, et en particulier l'analyse géologique et archéologique de Lartet [2], va contribuer à résoudre.

Lartet va en effet démontrer la présence dans une même couche géologique de mobiliers archéologiques, d'outils en pierre taillée et de restes humains. Il apporte ainsi la preuve irréfutable de l'ancienneté de l'Homme. Jusqu'au début du $\mathrm{XX}^{\mathrm{e}}$ siècle, cette preuve archéologique et le contexte chronologique des fossiles de Cro-Magnon feront néanmoins l'objet de discussions récurrentes et ne seront définitivement tranchés que lors de la publication de datations absolues des ossements humains [4] qui donneront définitivement raison à Lartet et Broca.

L'analyse géologique de Lartet [2] et l'étude anthropométrique de Broca [5] vont néanmoins installer les 7 squelettes de l'Abri de Cro-Magnon dans le panthéon paléoanthropologique. En effet, l'interprétation anthropologique 
de ces restes humains est dépendante de leur datation, et les conclusions de Broca sur la morphologie de cette « race des Eyzies » $[5: 352]$ ne peuvent être comprises que dans le contexte de la preuve apportée sur l'ancienneté des squelettes.

Cent cinquante ans plus tard, les Bulletins et Mémoires de la Société d'Anthropologie de Paris proposent dans ce numéro thématique, la réédition des deux publications originales qui présentèrent le site [2] et les restes humains [5] dans une version modernisée qui facilitera le travail de tout chercheur souhaitant utiliser ces références dans le cadre de ses recherches. Ces rééditions sont accompagnées de contributions originales visant à la fois à contextualiser historiquement cette découverte [1], à replacer le site de Cro-Magnon dans sa contribution scientifique à la Préhistoire [6] et à souligner son impact sur l'institution qui conserve les fossiles depuis 150 ans : le Musée de l'Homme [7]. Enfin, les recherches récentes sur les populations que nous appelons désormais « gravettiennes » continuent et connaissent un intérêt jamais démenti en paléoanthropologie. Les pratiques symboliques de ces hommes et leur mode de vie [8] sont, encore de nos jours, disséqués par les chercheurs. Les traces qu'ils nous ont laissées en France et ailleurs [9,10], ne cessent d'interpeller les scientifiques.

\section{Références}

Hurel A (2018) 1868 : Le moment Cro-Magnon. BMSAP 30(3-4) : $111-120$

Lartet L (1868) Une sépulture des troglodytes du Périgord. BMSAP II (3): $335-49$

Darwin C (1859) On The origin of species by means of natural selection, or the preservation of favoured races in the struggle for life. John Murray, London, 502p

Henry-Gambier D (2002) Les fossiles de Cro-Magnon (Les Eyziesde-Tayac, Dordogne), Nouvelles données sur leur position chronologique et leur attribution culturelle. BMSAP 14: 89-112

Broca P (1868) Sur les crânes et ossements des Eyzies. BMSAP II (3):350-92

Villotte S, Balzeau A (2018) Que reste-t-il des Hommes de CroMagnon 150 ans après leur découverte ? BMSAP 30(3-4): 146-152

Charrier-Arrighi N, Edy E, Fileyssant E (2018) Comment la bibliothèque Yvonne-Oddon rend-elle hommage à Cro-Magnon ? BMSAP 30(3-4): 192-195

Villotte S, Ogden A R, Trinkaus E (2018) Dental Abnormalities and Oral Pathology of the Pataud 1 Upper Paleolithic Human. BMSAP 30(3-4): 153-161

Villotte S (2018) Unexpected discovery of more elements from the Prehistoric immature skeleton from Baousso da Torre (Bausu da Ture) (Liguria, Italy). BMSAP 30(3-4): 162-168

Prat S, Péan S, Crépin L, Puaud S, Drucker D G, LázničkováGaletová M, Van der Plicht J, Valladas H, Verna C, Pathou-Mathis M, Lebon M, Yanevich A (2018) First anatomically modern humans from south-eastern Europe. Contributions of Buran-Kaya III site (Crimea). BMSAP 30(3-4): 169-179 